\title{
Component-Based Algebraic Specification and Verification in CafeOBJ
}

\author{
Răzvan Diaconescu* ${ }^{\star}$ Kokichi Futatsugi, and Shusaku Iida \\ Japan Advanced Institute of Science and Technology
}

\begin{abstract}
We present a formal method for component-based system specification and verification which is based on the new algebraic specification language CafeOBJ, which is a modern successor of OBJ incorporating several new developments in algebraic specification theory and practice.

We first give an overview of the main features of CafeOBJ, including its logical foundations, and then we focus on the behavioural specification paradigm in CafeOBJ, surveying the object-oriented CafeOBJ specification and verification methodology based on behavioural abstraction.

The last part of this paper further focuses on a component-based behavioural specification and verification methodology which features high reusability of both specification code and verification proof scores. This methodology constitutes the basis for an industrial strength formal method around CafeOBJ.
\end{abstract}

\section{Overview of CafeOBJ}

CafeOBJ (whose definition is given by [7]) is a modern successor of the OBJ language [18, 10] incorporating several new major developments in algebraic specification theory and practice. It is aimed to be an industrial strength language, suitable both for researchers and for practitioners. This section is devoted to a brief overview of CafeOBJ, including its main features, its specification and verification environment, and its logical foundations.

\subsection{CafeOBJ Main Features}

Equational Specification and Programming. This is inherited from OBJ [18, 10] and constitutes the basis of the language, the other features being somehow built on top of it. As with OBJ, CafeOBJ is executable (by term rewriting), which gives an elegant declarative way of functional programming, often referred as algebraic programming 1 As with OBJ, CafeOBJ also permits equational specification modulo several equational theories such as associativity, commutativity, identity, idempotence, and combinations between all these. This feature is reflected at the execution level by term rewriting modulo such equational theories.

\footnotetext{
* On leave from the Institute of Mathematics of the Romanian Academy, PO Box 1-764, Bucharest 70700, ROMANIA.

${ }^{1}$ Please notice that although this paradigm may be used as programming, this aspect is still secondary to its specification side.
} 
Behavioural Specification. Behavioural specification [13, 14, 4] provides another novel generalization of ordinary algebraic specification but in a different direction. Behavioural specification characterizes how objects (and systems) behave, not how they are implemented. This new form of abstraction can be very powerful in the specification and verification of software systems since it naturally embeds other useful paradigms such as concurrency, object-orientation, constraints, nondeterminism, etc. (see [14] for details). Behavioural abstraction is achieved by using specification with hidden sorts and a behavioural concept of satisfaction based on the idea of indistinguishability of states that are observationally the same, which also generalizes process algebra and transition systems (see [14]).

CafeOBJ directly supports behavioural specification and its proof theory through special language constructs, such as

- hidden sorts (for states of systems),

- behavioural operations (for direct "actions" and "observations" on states of systems),

- behavioural coherence declarations for (non-behavioural) operations (which might be either derived (indirect) "observations" or "constructors" on states of systems), and

- behavioural axioms (stating behavioural satisfaction).

The advanced coinduction proof method receives support in CafeOBJ via a default (candidate) coinduction relation (denoted $=^{*}=$ ). In CafeOBJ, coinduction can be used either in the classical HSA sense [14] for proving behavioural equivalence of states of objects, or for proving behavioural transitions (which appear when applying behavioural abstraction to RWL) 2

Besides language constructs, CafeOBJ supports behavioural specification and verification by several methodologies 3 CafeOBJ currently highlights a methodology for concurrent object composition which features high reusability not only of specification code but also of verifications [7, 8]. Behavioural specification in CafeOBJ might also be effectively used as an object-oriented (state-oriented) alternative for traditional ADT specifications. Experiments seem to indicate that an object-oriented style of specification even of basic data types (such as sets, lists, etc.) might lead to higher simplicity of code and drastic simplification of verification process [7].

Behavioural specification is reflected at the execution level by the concept of behavioural rewriting [7, 4] which refines ordinary rewriting with a condition ensuring the correctness of the use of behavioural equations in proving strict equalities.

Rewriting Logic Specification. Rewriting logic specification in CafeOBJ is based on a simplified version of Meseguer's rewriting logic [20] specification framework for concurrent systems which gives a non-trivial extension of traditional algebraic specification towards concurrency. RWL incorporates many different models of concurrency

\footnotetext{
${ }^{2}$ However, until the time this paper was written, the latter has not been yet explored sufficiently, especially practically.

${ }^{3}$ This is still an open research topic, the current methodologies might be developed further and new methodologies might be added in the future.
} 
in a natural, simple, and elegant way, thus giving CafeOBJ a wide range of applications. Unlike Maude [2], the current CafeOBJ design does not fully support labelled RWL which permits full reasoning about multiple transitions between states (or system configurations), but provides proof support for reasoning about the existence of transitions between states (or configurations) of concurrent systems via a built-in predicate (denoted $==>$ ) with dynamic definition encoding both the proof theory of RWL and the user defined transitions (rules) into equational logic.

From a methodological perspective, CafeOBJ develops the use of RWL transitions for specifying and verifying the properties of declarative encoding of algorithms (see [7]) as well as for specifying and verifying transition systems.

Module System. The principles of the CafeOBJ module system are inherited from OBJ which builds on ideas first realized in the language Clear [1], most notably institutions [11,9]. CafeOBJ module system features

- several kinds of imports,

- sharing for multiple imports,

- parameterized programming allowing

- multiple parameters,

- views for parameter instantiation,

- integration of CafeOBJ specifications with executable code in a lower level language

- module expressions.

However, the theory supporting the CafeOBJ module system represents an updating of the original Clear/OBJ concepts to the more sophisticated situation of multi-paradigm systems involving theory morphisms across institution embeddings [5], and the concrete design of the language revise the OBJ view on importation modes and parameters [7].

Type System and Partiality. CafeOBJ has a type system that allows subtypes based on order sorted algebra (abbreviated OSA) [17, 12]. This provides a mathematically rigorous form of runtime type checking and error handling, giving CafeOBJ a syntactic flexibility comparable to that of untyped languages, while preserving all the advantages of strong typing.

Since at this moment there are many order sortedness formalisms, many of them very little different from others, and each of them having its own technical advantages and disadvantages and being most appropriate for a certain class of applications, we decided to keep the concrete order sortedness formalism open at least at the level of the language definition. Instead we formulate some basic simple conditions which any concrete CafeOBJ order sorted formalism should obey. These conditions come close to

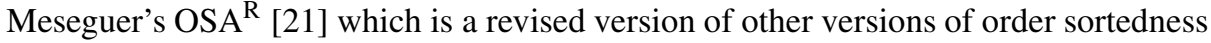
existing in the literature, most notably Goguen's OSA [12].

CafeOBJ does not directly do partial operations but rather handles them by using error sorts and a sort membership predicate in the style of membership equational logic (abbreviated MEL) [21]. The semantics of specifications with partial operations is given by MEL. 


\subsection{The CafeOBJ Specification and Verification Environment}

Although this is rather a feature of the current system rather than of the language, due to its importance for the effective use of the current CafeOBJ system, we briefly survey it here.

The CafeOBJ system includes an environment supporting specification documents with formal contents over networks and enabling formal verifications of specifications. The CafeOBJ environment takes advantage of current InterNet technologies and can be thought as consisting of four parts:

- The interpreter in isolation acts very much like the OBJ3 interpreter by checking syntax and evaluating (reducing) terms. In addition, the CafeOBJ interpreter incorporates an abstract TRS machine and a compiler.

- The proof assistant extends the theorem proving capabilities of the interpreter with more powerful, dedicated provers.

- The document manager takes care of processing of specification documents over networks.

- Specification libraries focus on several specific problem domains, such as objectoriented programming, database management, interactive systems, etc.

\subsection{CafeOBJ Logical Foundations}

CafeOBJ is a declarative language with firm mathematical and logical foundations in the same way as other OBJ-family languages (OBJ, Eqlog [15, 3], FOOPs [16], Maude [20]) are. The reference paper for the CafeOBJ mathematical foundations is [6], while the book [7] gives a somehow less mathematical easy-to-read (including many examples) presentation of the semantics of CafeOBJ. In this section we give a very brief overview of the CafeOBJ logical and mathematical foundations, for a full understanding of this aspect of CafeOBJ the reader is referred to [6] and [7].

The mathematical semantics of CafeOBJ is based on state-of-the-art algebraic specification concepts and results, and is strongly based on category theory and the theory of institutions [11, 5, 9]. The following are the principles governing the logical and mathematical foundations of CafeOBJ:

P1. there is an underlying logid 4 in which all basic constructs and features of the language can be rigorously explained.

P2. provide an integrated, cohesive, and unitary approach to the semantics of specification in-the-small and in-the-large.

P3. develop all ingredients (concepts, results, etc.) at the highest appropriate level of abstraction.

The CafeOBJ Cube. CafeOBJ is a multi-paradigm language. Each of the main paradigms implemented in CafeOBJ is rigorously based on some underlying logic; the paradigms resulting from various combinations are based on the combination of

\footnotetext{
${ }^{4}$ Here "logic" should be understood in the modern relativistic sense of "institution" which provides a mathematical definition for a logic (see [11]) rather than in the more classical sense.
} 
logics. The structure of these logics is shown by the following CafeOBJ cube, where the arrows mean embedding between the logics, which correspond to institution embeddings (i.e., a strong form of institution morphisms of [11,9]) (the orientation of arrows correspond to embedding "less complex" into "more complex" logics).

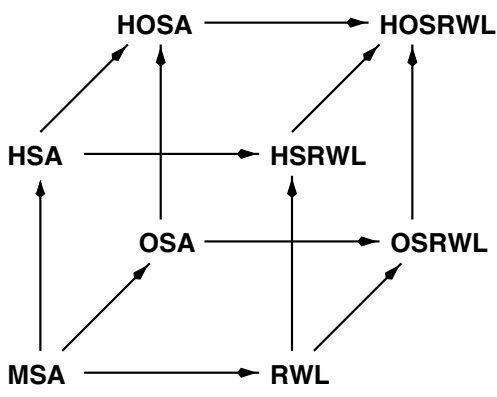

$$
\begin{aligned}
& \mathrm{H}=\text { hidden } \\
& \mathrm{A}=\text { algebra } \\
& \mathrm{O}=\text { order } \\
& \mathrm{S}=\text { sorted } \\
& \mathrm{M}=\text { many } \\
& \mathrm{RWL}=\text { rewriting logic }
\end{aligned}
$$

The mathematical structure represented by this cube is that of a lattice of institution embeddings [5, 6]. By employing other logical-based paradigms the CafeOBJ cube may be thought as a hyper-cube (see [6, 7] for details). It is important to understand that th CafeOBJ logical foundations are based on the CafeOBJ cube rather than on its flattening represented by HOSRWL 5

The design of CafeOBJ lead to several important developments in algebraic specification theory. One of them is the concept of extra theory morphism [5], which is a concept of theory morphism across institution embeddings, generalizing the ordinary (intra) theory morphisms to the multi-paradigm situation. Another important theoretical development is constituted by the formalism underlying behavioural specification in CafeOBJ which is a non-trivial extension of classical hidden algebra [14] in several directions, most notably permitting operations with several hidden arguments via the crucial coherence property. This extension is called "coherent hidden algebra" in [4] and comes very close to the "observational logic" of Bidoit and Hennicker [19]. The details of the "coherent hidden algebra" institution can be found in [6].

\section{Behavioural Specification in CafeOBJ}

Behavioural specification might be the most distinctive feature of CafeOBJ within the broad family of algebraic specification languages. As mentioned above, behavioural specification paradigm is incorporated into the design of the language in a rather direct way. Also, this paradigm constitutes the core of the current CafeOBJ object-oriented specification and verification methodologies. We devote this section to a methodological presentation of the behavioural specification paradigm in CafeOBJ, trying also to explain the main concepts behind this paradigm.

\footnotetext{
${ }^{5}$ The technical reason for this is that model reducts across some of the edges (i.e., the leftto-right ones) of the CafeOBJ cube involve both an ordinary model reduct and a non-trivial reduct along the corresponding institution embedding, see [6, 5, 7] for details.
} 


\subsection{Basic Behavioural Specification}

Basic behavioural specification is the simplest level of behavioural specification in which the operations are either actions or observations on the states of the objects. Let us consider an object-oriented (or "state-oriented") CafeOBJ specification for lists:

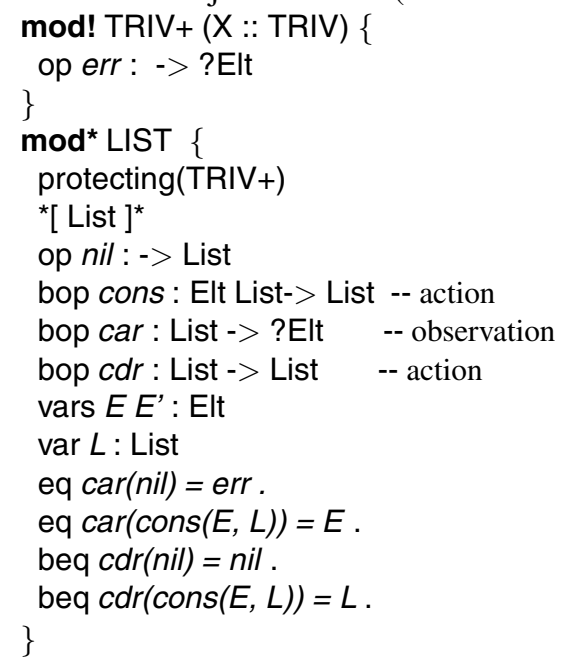

This is quite different from the usual data-oriented specification of lists. In our behavioural specification, lists are treated as objects with states (the sort of states is the hidden sort List), and the usual list operations (cons and cdr) act on the states of the list object or (car) observe the states. Actions and observations are specified as behavioural operations. In general, a behavioural operation is called action iff its sort is hidden (i.e., state type), and is called observation iff its sort is visible (i.e., data type). Behavioural operations are restricted to have exactly one hidden sort in their arity, this monadicity property being characteristic to behavioural operations (either actions or observations). Behavioural operations define the behavioural equivalence relation between the states of the object, denoted as $\sim$ :

$$
s \sim s^{\prime} \text { iff }\left[c(s)=c\left(s^{\prime}\right) \text { for all visible behavioural contexts } c\right]
$$

A behavioural context $c$ is any string of behavioural operations (this makes sense because of the monadicity property on hidden sorts of the behavioural operations). $c$ is visible iff its sort is visible; this is the same as saying that $c$ has an observation at the top. It is important to notice that behavioural equivalence is a semantic notion; this means that whenever we consider a behavioural equivalence relation we need to consider a model (i.e., an implementation) for the specification?

CafeOBJ methodologies introduce a graphical notation extending the classical ADJdiagram notation for data types for behavioural specification in which

G1. Sorts are represented by ellipsoidal disks with visible (data) sorts represented in white and hidden (state) sorts represented in grey, and with subsort inclusion represented by disk inclusion, and

\footnotetext{
${ }^{6}$ Which needs not to be a concrete one.
} 
G2. Operations are represented by multi-source arrows with the monadic part from the hidden sort thickened in case of behavioural operations.

The list specification can be therefore visualised as follows:

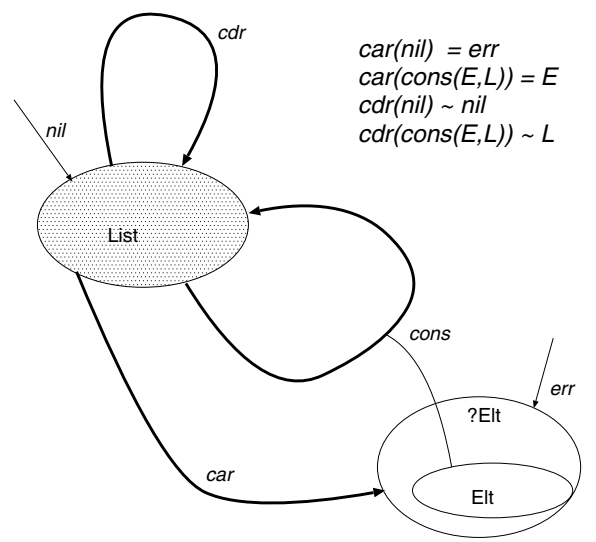

Several other aspects of this specifications need special attention. The first one concerns the data of this specification and the error handling aspect of this methodology. LIST specifies a list object over any set of elements. "Any set of elements" is specified by the built-in module TRIV which specifies one sort (Elt) with loose denotation (hence its denotation is given by all sets); this is used as a parameter of the specification LIST and can be instantiated to any concrete data type. The error handling aspect arises because of the partiality of car. TRIV+ just introduces a new error element (err). The error supersort ?Elt is built-in 7 and err is the only new element belonging to [the denotation of] ?Elt; this is ensured by the free extension of [the loose denotation of] TRIV which is specified by giving TRIV+ initial denotation (mod!). Notice that this style of error handling contrasts the more complex data-oriented approach which uses a subsort for the non-empty lists and overloads the list operations on this subsort. This methodological simplification is mainly possible because of the loose denotation of behavioural specification (with the adequate "loose" behavioural equality) which avoids the strictness of the initial denotation of the data-oriented approach.

Another aspect is given by the use of behavioural equations in the specification LIST. Behavioural equations represent behavioural equivalence relations between states rather than strict equalities. Therefore each model (implementation) of LIST does not need to interpret $c d r(\operatorname{cons}(e, l))$ as $l$, where $e$ is an element and $l$ is a lis $\underline{8}$, but rather as a state behavioural equivalent to $l$. For example, if one implements the list object as an array with pointer, in this model (implementation) this equality does not hold strictly, but it holds behaviourally. Generally speaking, behavioural equality is the meaningful equality on hidden sorts, while the strict equality is the meaningful equality for the visible (data) sorts. However, there are situations when the strict equality on hidden sorts is also necessary. Behavioural abstraction also provides a nice way of error handling for

\footnotetext{
${ }^{7}$ It is provided by the system.

${ }^{8}$ Better said, a state of the list object.
} 
hidden sorts, as shown by the other behavioural equation. Thus instead of introducing a (hidden) error for $c d r(n i l)$, we rather shift the error handling to the data type by saying this is behaviourally equivalent to nil9 A finer analysis of the behavioural equivalence on the list object (see the section below) tells us that the behavioural equality between $c d r(n i l)$ and $n i l$ is exactly the same with saying that $\operatorname{car}\left(c d r^{n}(n i l)\right)=$ err for all natural numbers $n$, which is the natural minimal condition for the behaviour of nil.

\subsection{Behavioural Specification with Hidden Constructors}

Behavioural specification with hidden constructors is a more advanced level of behavioural specification which relies on the important novel concept of behavioural coherence first defined and studied in [7. 4] and which was first realized by the CafeOBJ language [7].

At the general level, a hidden constructor is an operation on hidden sort 10 whose sort is also hidden and which is not declared behavioural. This means that such operation does not take part in the definition of the behavioural equivalence relation. Also (and related to the above), a hidden constructor need not be monadic on the hidden sorts, thus it may admit several hidden sorts in the arity.

In the data-oriented specification of lists there is a difference in nature between cons and $c d r$, in that cons is a "constructor" and $c d r$ is a "destructor". This different nature of cons and cdr reflects in the behavioural specification too and is formally supported by the fact that one may prove (from the specification LIST) that for all lists $l$ and $l^{\prime}$,

$$
l \sim l^{\prime} \text { iff }\left[\operatorname{car}\left(c d r^{n}(l)\right)=\operatorname{car}\left(c d r^{n}\left(l^{\prime}\right)\right) \text { for all natural numbers } n\right]
$$

Technically this means that for the purpose of defining the appropriate behavioural equivalence for lists, cons does not play any rôle, therefore it may be specified as an ordinary operation, hence cons is a hidden constructor. Consequently, the only real behavioural operations are the observation car and the action cdr. This new specification for lists can be visualized by the following CafeOBJ diagram:

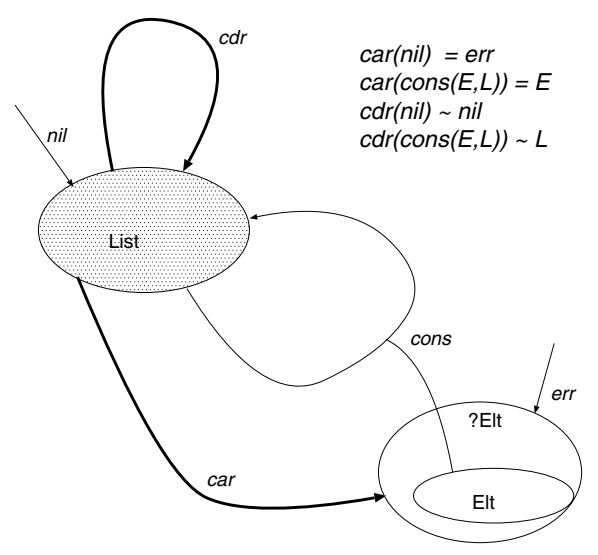

\footnotetext{
${ }^{9}$ Recall that in LISP $c d r($ nil) is also equal to nil but under a LISP concept of equality; it may be worthwhile trying to think LISP equality in behavioural abstraction terms.

${ }^{10}$ Which may also have visible sorts in the arity.
} 
This "neutrality" of cons with respect to the behavioural equivalence may be understood by the fact that cons preserves the behavioural equivalence defined by $c d r$ and car only. This basic property of hidden constructors is called coherence [7, 4], which in general means the preservation of the behavioural equivalence relation by the hidden constructors. In CafeOBJ the coherence property is user specified as an operation attribute:

op cons : Elt List $->$ List $\quad$ coherent

The semantic meaning of a coherence declaration is that the corresponding specification admits only models for which the operation is coherent (i.e., it preserves the behavioural equivalence). For methodological reason CafeOBJ admits potentially noncoherent operations (in the absence of the coherence declaration), however in the final version of the specification all hidden constructors should be declare coherent both for semantical and operational reasons 11

\subsection{Behavioural Coherence Methodologies}

In the above list example the coherence of cons can be proved as a formal property of the specification 12. This means that in any model of this specification the interpretation of cons automatically preserves the behavioural equivalence, so the class of models (implementations) of the specification with cons not specified as coherent coincides with its subclass of models for the case when cons is specified as coherent. Such constructors, which occurs frequently and which are practically desirable are called the conservative.

The opposite case is represented by the non-conservative constructors, which corresponds to the situation when the class of models for the case when the operation is specified as coherent is a strict subclass of the class of models when the operation is not specified as coherent. Proof-theoretically, this means the coherence property of the operation cannot be formally proved as a consequence property of the [rest of the] specification. Because of its semantical aspect, the methodology of non-conservative constructors is more advanced and sophisticated than the conservative one. However it might be very useful in dealing with non-terminating computations, in a way similar to the the use of commutativity attribute for operations in classical algebraic specification (see [4] for more details).

Proving Behavioural Coherence. We now concentrate to an example illustrating the behavioural coherence methodology of conservative constructors. Consider the following behavioural specification of sets:

${ }^{11}$ A simple example is given by the coherence proof scores, when one needs to start with the absence of the coherence declaration for the operation. The coherence declaration is added to the specification only after its proof.

12 The CafeOBJ proof score for this is rather simple; we leave it as exercise for the reader. 


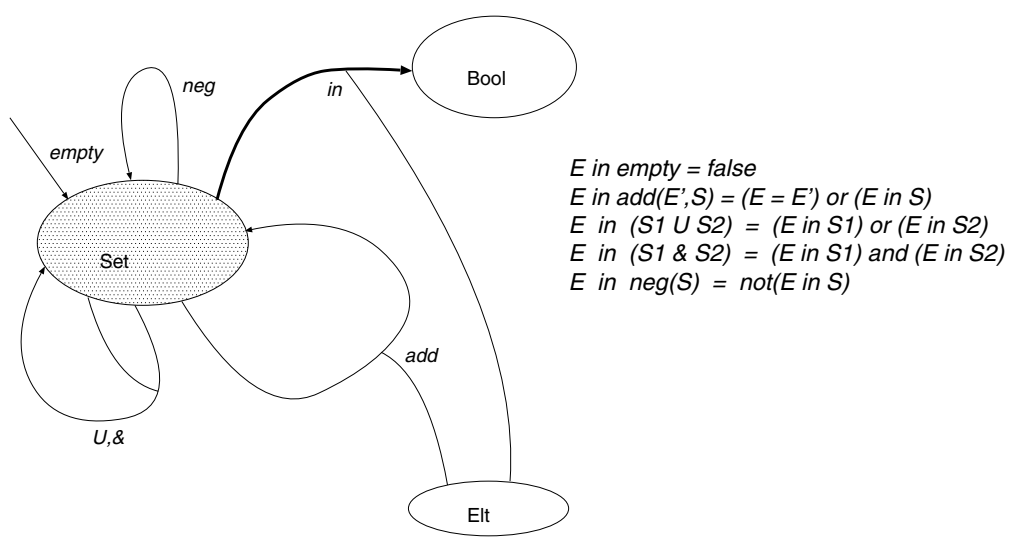

This specification has only one behavioural operation, namely the observation _in_. The hidden constructors $a d d, U_{-}, \mathcal{E}_{-}$, and neg can be proved coherent by the following CafeOBJ proof score:

open .

ops s1 s2 s1's2' : -> Set .-- arbitrary sets as temporary constants

ops $e e^{\prime}:->$ Elt . -- arbitrary elements as temporary constants

ceq $S 1=^{*}=S 2=$ true if $(e$ in $S 1)==(e$ in $S 2)$.- definition of behavioural equivalence beq $s 1=s 1^{\prime}$. -- hypothesis

beq $s 2=s 2^{\prime}$. -- hypothesis

red $\operatorname{add}(e, s 1)={ }^{*}=\operatorname{add}\left(e, s 1^{\prime}\right)$. -- beh coherence of $\operatorname{add}(-)$ for variable clash at Elt

red $\operatorname{add}\left(e^{\prime}, s 1\right)={ }^{*}=\operatorname{add}\left(e^{\prime}, s 1^{\prime}\right) .-$ beh coherence of $\operatorname{add}(-)$ for no variable clash at Elt

red $(s 1 \cup s 2)={ }^{*}=\left(s 1^{\prime} \cup s 2^{\prime}\right)$. -- beh coherence of _ $U_{-}$

red $(s 1 \& s 2)={ }^{*}=\left(s 1^{\prime} \& s 2^{\prime}\right)$. -- beh coherence of _\&_

red $n e g(s 1)=^{*}=n e g\left(s 1^{\prime}\right)$. -- beh coherence of neg_

close

where

Notice the simplicity of this proof score which uses the built-in default coinduction relation $=^{*}=$ which in practice is oftenly the behavioural equivalence. Once the coherence of the hidden constructors is formally proved, their coherence declarations are added to the specification, thus obtaining the final version of the specification under the methodology of conservative hidden constructors.

\subsection{Behavioural Verification}

One of the great advantages of behavioural specification lies in the simplicity of the verification stage which sometimes contrasts sharply with the complexity of corresponding data type verifications. Sets are one of the examples showing clearly the greater simplicity of behavioural verifications. While the verification of set-theoretic properties in the data approach gets into a very complex induction process, behavioural properties of sets can be proved almost immediately. The following is the very simple CafeOBJ proof score for one of De Morgan laws:

open .

op e : -> Elt . 
ops s1 s2 s3: -> Set .

-- definition of behavioural equivalence

ceq $S 1:$ Set $={ }^{*}=S 2:$ Set $=$ true if $(e$ in S1) $==(e$ in S2) .

red $n e g(s 1 \cup s 2)={ }^{*}=(n e g(s 1) \& n e g(s 2))$. -- proof of de Morgan law

close

Notice that CafeOBJ proof scores follow a certain pattern. Firstly, one "opens" an working module for adding temporary entities; this is the command open. The command close "closes" the module, thus resetting it to its original content. After opening, one introduces temporary constants which, due to the fact that they are arbitrary, have the meaning of variables local to the proof score. The next step consists of setting up the proof context, involving declaring the hypotheses, etc. The proofs are effectively done by CafeOBJ reduction mechanism (the command red). The inputs of red are usually logical atomic properties which are encoded as Boolean terms, so in general one expects a true answer from the CafeOBJ interpreter for each of such reductions. In case of more complex proofs, a CafeOBJ proof score flatten the corresponding proof tree to a sequence of hypotheses and reductions. In this flattening, properties proved by reductions might be lemmas which might be later used as hypotheses for other reductions.

Behavioural Rewriting. The execution of behavioural specifications is done by behavioural rewriting, which is a refinement of ordinary (term) rewriting that ensures the correctness of rewriting when using behavioural equations as rewrite rules. The basic condition of behavioural rewriting requires the existence of a path formed by behavioural or coherent operations on top of the redex. When inferring strict equalities, it is required in addition that the top of such path is of visible sort. For example, when proving the behavioural coherence of add,

$\operatorname{red} \operatorname{add}(e, s 1)={ }^{*}=\operatorname{add}\left(e, s 1^{\prime}\right)$.

means a strict equality reduction. In this case the first behavioural equation of the corresponding proof score cannot be used as a first rewriting step since the condition of behavioural rewriting is not fulfilled. This triggers the use of the conditional equation instead as a first rewriting step, and only after this the use of behavioural equations of the proof score fall under the required condition.

\subsection{Behavioural Refinement}

Object refinement in behavioural specification is a relaxed form of behavioural specification morphism (see [7] for more details). As an example we show how behavioural lists refine behavioural sets, which corresponds to the basic intuition of sets implemented as lists. For simplicity of presentation we consider here only the case of basic sets, without union, intersection, and negation 13 . The refinement of behavioural basic sets to lists was represented above by extending the graphical notation previously introduced with:

G3. Refinement of sorts and operations is written by $\lrcorner_{-}$and sharing the same figure (disk or arrow) in the diagram.

G4. Newly introduced sorts and operations are represented by dotted lines.

$\overline{13}$ Our example can be easily extended to union and intersection, but not so easily to negation. 


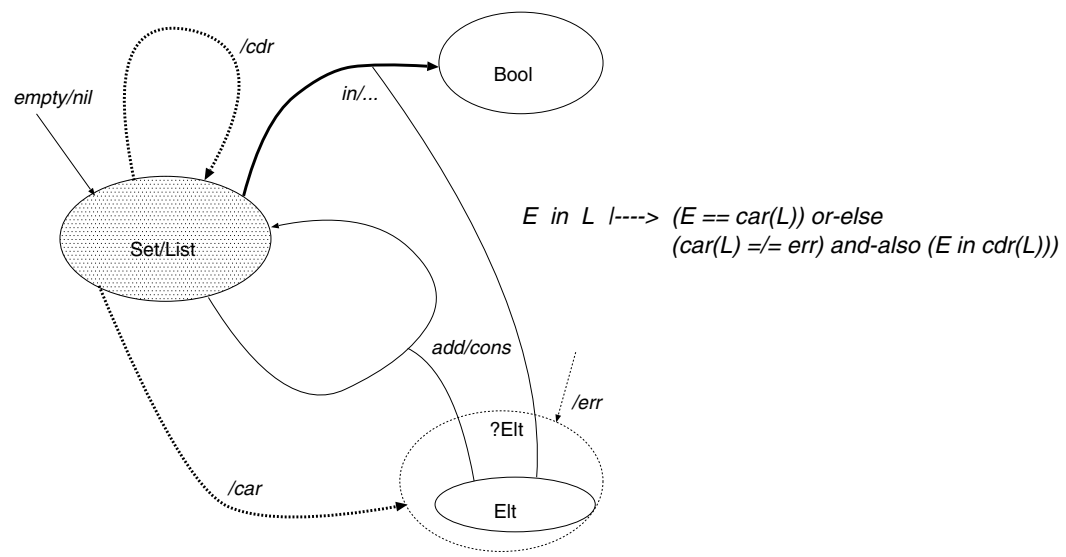

In this refinement, the hidden sort Set is refined to the hidden sort List (this means that any state of the set object and be implemented by a state of the list object), add is refined to cons. The list object has the observation car and the action cdr as new behavioural operations and also adds the error handling. The set object observation _in_ is refined to a derived observation (using some operational versions of the Boolean connectives). This refinement can be encoded in CafeOBJ by the following module import:

mod $^{*}$ LIST' $\{$ protecting(LIST)

op_in_: Elt List -> Bool $\{$ coherent $\}$-- coherence provable from the rest of spec vars $E E^{\prime}:$ Elt

$\operatorname{var} L:$ List

eq $E$ in $L=(E==\operatorname{car}(L))$ or-else $(\operatorname{car}(L)=/=$ err and-also $E$ in $\operatorname{cdr}(L))$. $\}$

The following is the proof score for the fact that the mapping defined above is indeed a refinement, i.e., the property of add holds for cons:14

open LIST'.

ops e e1 e2 : -> Elt . -- arbitrary elements as temporary constants

op I: - > List . -- arbitrary list as temporary constant

eq 1 in I = true . -- the basic case when the element does belong to the list

eq e2 in I = false . -- the basic case when the element does not belong to the list

red $e$ in nil $==$ false. -- the nil case

red $e 1$ in $\operatorname{cons}(e, l)==$ true .

red $e 2$ in $\operatorname{cons}(e, l)==$ false .

red $e$ in $\operatorname{cons}(e, l)==$ true .-- the element clash case

close

\section{Concurrent Object Composition in CafeOBJ}

In this section we present the object composition method of CafeOBJ based on the behavioural specification paradigm. We present here a simplified method which does not use behavioural coherence. We use UML to represent object composition:

\footnotetext{
14 This involves a small case analysis.
} 


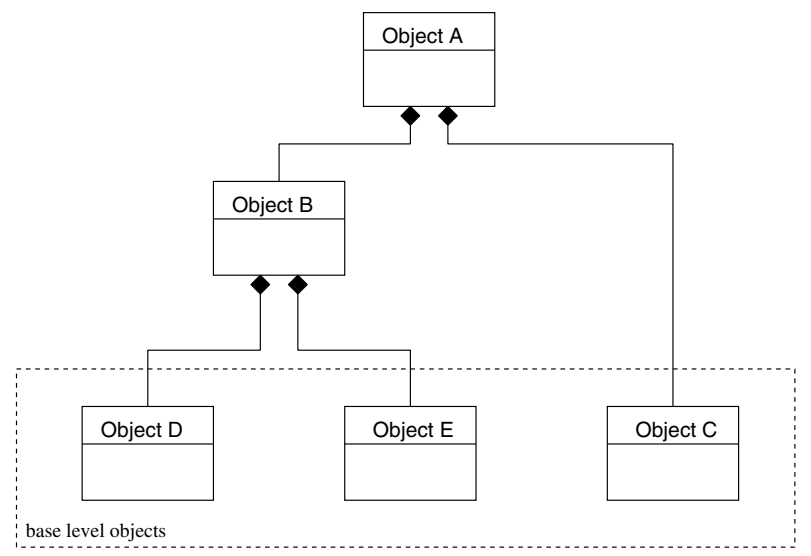

In the above UML figure, $B$ is composed of $D$ and $E, A$ of $B$ and $C$, and noncompound objects (i.e., objects with no components) are called base level objects. A composition in UML is represented by line tipped by a diamond, and if necessary, qualified by the numbers of components ( $\mathbf{1}$ for one and * for many).

Projection operations from the hidden sort of the states of the compound object to the hidden sorts of the states of the component objects constitute the main technical concept underlying the CafeOBJ composition method; projection operations are related to the lines of UML figures. Projection operations are subject to the following mathematical conditions [8,7]. Given a compound object $O$ (with signature $(V \cup H, \Sigma)$ for which $V$ is a set of visible sorts and $H$ is a set of hidden sorts), a set of the labels of its components $O b j$, and the components $\left\{O_{n}\right\}_{n \in O b j}$ of $O$ (with signatures $\left(V_{n} \cup H_{n}, \Sigma_{n}\right)$ ), the projection operations $\pi_{n}: h \rightarrow h_{n}$ (with $h \in H$ and $h_{n} \in H_{n}$ ) for each $n \in O b j$ satisfy the following conditions:

1. for each observation $o$ of $O$, there exists $n \in O b j$ and a data (possibly derived) operation $f: v_{n_{1}} \ldots v_{n_{i}} \rightarrow v\left(v_{n_{i}} \in V_{n}\right.$ and $v \in V$ where $i$ is a natural number), and for each $n_{k}$ with $k \in\{1, \ldots, i\}$ a visible $O_{n_{k}}$-context $c_{n_{k}}$ (i.e., made only of operations of the signature of $\left.O_{n_{k}}\right)$ such that $o(X)=f\left(\left(c_{n_{1}} \circ \pi_{n_{1}}\right)(X), \ldots,\left(c_{n_{i}} \circ \pi_{n_{i}}\right)(X)\right)$ for all states $X$,

2. for each action $a$ of $O$, and for each $n \in O b j$, there exists a sequence of actions $a_{n}$ such that $\pi_{n} \circ a=a_{n} \circ \pi_{n}$, and

3. for each constant const of $O$, and for each $n \in O b j$, there exists a constant const $_{n}$ such that $\pi_{n}($ const $)=$ const $_{n}$ (i.e., the initial state of a compound object should be is related to the initial state of each component.)

In the compound objects we only define communication between the components; this means that the only equations at the level of the specification of the compound objects are the ones relating the actions and observations of the compound objects to those of the components as described above. In the case of synchronized compositions, the equations of the previous definition are conditional rather than unconditional. Their conditions are subject to the following: 
- each condition is a finite conjunction of equalities between terms of the form $c_{n} \circ \pi_{n}$ (where $\pi_{n}$ is a projection operator and $c_{n}$ is an $O_{n}$-context) and terms in the data signature, and

- disjunction of all the conditions corresponding to a given left hand side (of equations regarded as a rewrite rule) is true.

\subsection{Parallel Connection}

The components of a composite object are connected (unsynchronized) in parallel if there is no synchronization between them. In order to define the concept of synchronization, we have to introduce the concept of action group. Two actions of a compound object are in the same action group when they change the state of the same component object via a projection operation. Synchronization appears when:

- there exists an overlapping between some action groups, or

- the projected state of the compound object (via a projection operation) depends on the state of a different (from the object corresponding to the projection operation) component.

The first case is sometimes called broadcasting and the second case is sometimes called client-server computing. In the unsynchronized case, we have full concurrency between all the components, which means that all the actions of the compound object can be applied concurrently, therefore the components can be implemented as distributed processes or concurrent processes with multi-thread which are based on asynchronous communications.

For unsynchronized parallel connection, we consider a bank account system example. Firstly, we consider a very simple bank account system which consists of a fixed numbers of individual accounts, lets actually consider the case of just two accounts. The specification of an account can be obtained just by renaming the specification COUNTER 1 of a counter object with integers as follows

mod $^{*}$ ACCOUNT1 $\left\{\right.$ protecting(COUNTER1 ${ }^{*}$ \{ hsort Counter $->$ Account 1 , op init-counter $->$ init-account1 $\})\}$

mod* ACCOUNT2 $\{$ protecting(COUNTER1 * $\{$ hsort Counter $->$ Account2, op init-counter -> init-account2 $\})\}$

where COUNTER1 is represented in CafeOBJ graphical notation as follows:

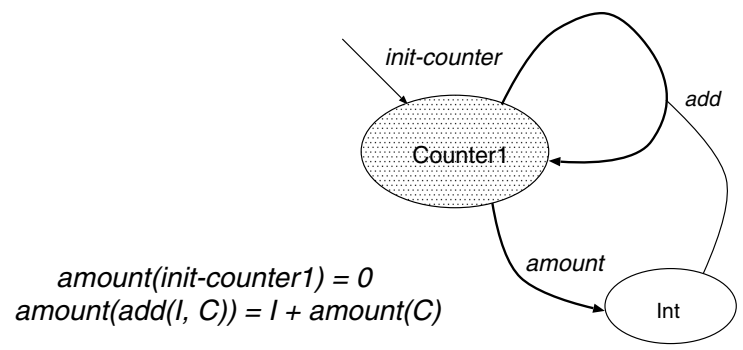


We then compose these two account objects as in the following double figure containing both the UML and the CafeOBJ graphica 15 representation of this composition, where deposit1 and withdraw1 are the actions for the first account, balance1 is the observation for the first account, account1 is the projection operation for the first account, and deposit2, withdraw2, balance2, and account2 are the corresponding actions, observation, and projection operation for the second account:
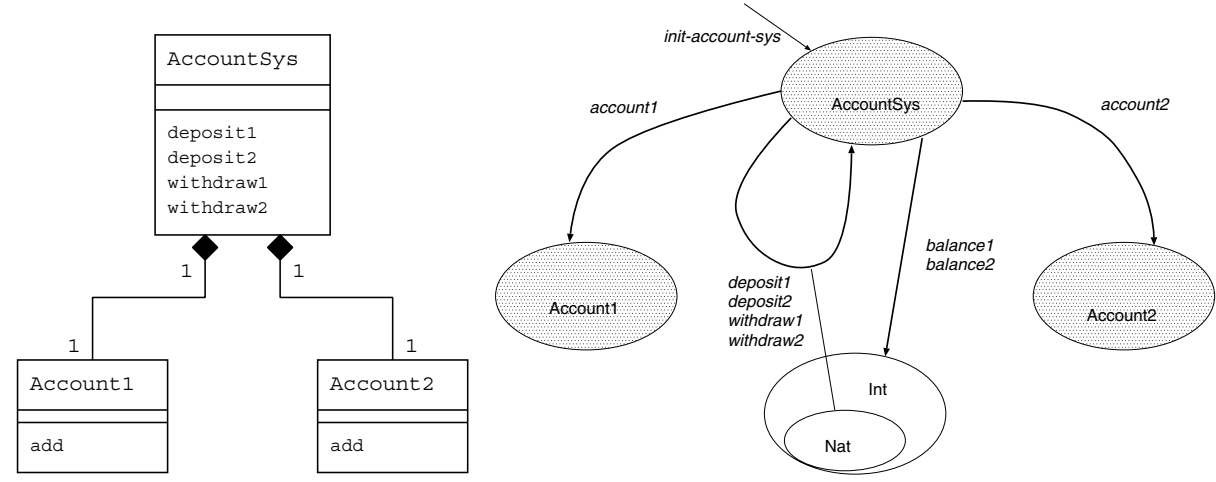

The equations for this parallel connection (composition) are as follows:

eq balance1 $(A S)=$ amount $($ account $1(A S))$.

eq balance2 $(A S)=$ amount $($ account2 $(A S))$.

eq account1 (init-account-sys) = init-account1 .

eq $\operatorname{account1}(\operatorname{deposit1}(N, A S))=\operatorname{add}(N, \operatorname{account1}(A S))$.

eq account1 (deposit2 $(N, A S))=\operatorname{account1}(A S)$.

eq account1 (withdraw1 $(N, A S))=\operatorname{add}(-(N)$, $\operatorname{account1}(A S))$.

eq account1 (withdraw2( $N, A S))=\operatorname{account1}(A S)$.

eq account2(init-account-sys) = init-account2 .

eq account2(deposit1 $(N, A S))=$ account2 $(A S)$.

eq account2(deposit2 $(N, A S))=\operatorname{add}(N, \operatorname{account2}(A S))$.

eq account2(withdraw1 $(N, A S))=$ account2(AS) .

eq account2(withdraw2 $(N, A S))=\operatorname{add}(-(N)$, account2(AS)) .

Notice that besides the first two equations relating the observations on the compound object to those on the components, the other equations relate the actions of the account system to the actions of the components. Remark that the actions corresponding to one component do not change the state of the second component (via the projection operation), hence this composition is unsynchronized. In fact these equations expressing the concurrency of composition need not be specified by the user, in their absence they may be generated internally by the system, thus reducing the specification of the composition to the essential information which should be provided by the user.

${ }^{15}$ The CafeOBJ graphical representation corresponds to the module defining this object composition rather than to the "flattened" specification, hence the operations of the components are not included in the figure. 


\subsection{Dynamic Connection}

In this subsection, we extend the previous bank account system example to support an arbitrary number of accounts. The accounts are created or deleted dynamically, so we call such architecture pattern dynamic connection and we call the objects connected dynamically as dynamic objects. A dynamic object has an object identifier type as the arity of its initial state (which is quite a natural idea that in object-oriented programming languages, language systems automatically providing a pointer for each object when created). We therefore firstly extend the specification of the counter to a dynamic object

op init-counter : Uld -> Counter

where Uld is a sort for user identifiers. The actions add-account and del-account maintain the user accounts. add-account creates accounts with some initial balance while del-account deletes the accounts; both of them are parameterized by the user identifiers Uld. Each of deposit and withdraw is also parameterized by the user identifiers. Most notably, the projection operation for Account is also parameterized by Uld. The initial state of AccountSys has no account, so it is mapped to the error state called no-account. The structure of the new bank account system can be represented in UML and CafeOBJ graphical notation as follows:
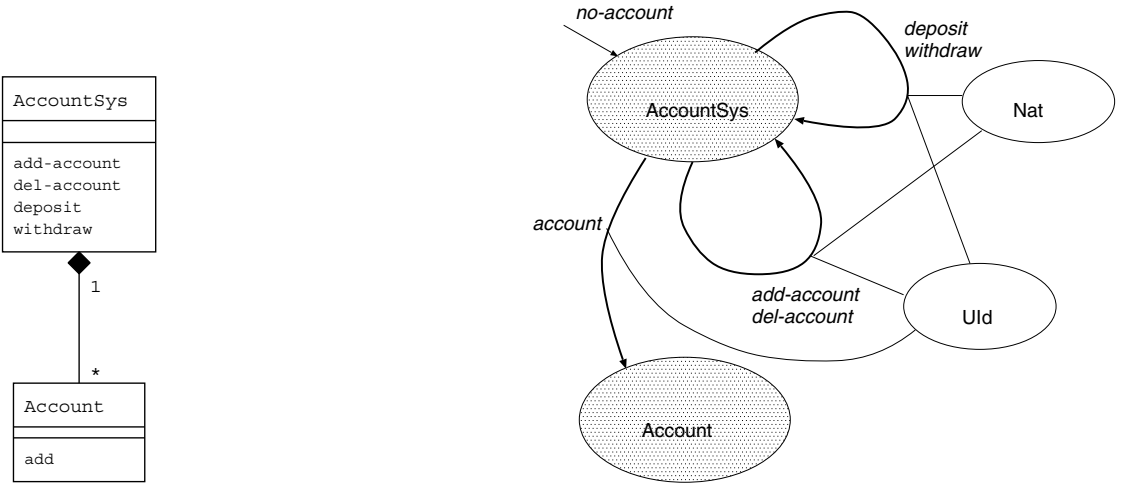

Finally, the equations relate the actions of AccountSys to those of Account via the projection operation only when they correspond to the specified user account. Here is the essential part of the CafeOBJ code for the dynamic system of accounts specification:

eq account $(U$, init-account-sys $)=$ no-account .

ceq $\operatorname{account}\left(U, \operatorname{add}\right.$-account $\left.\left(U^{\prime}, N, A\right)\right)=\operatorname{add}(N$, init-account $(U))$ if $U==U^{\prime}$.

ceq $\operatorname{account}\left(U, \operatorname{add}\right.$-account $\left.\left(U^{\prime}, N, A\right)\right)=\operatorname{account}(U, A)$ if $U=/=U^{\prime}$.

ceq account $(U$, del-account $(U, A))=$ no-account if $U==U^{\prime}$.

ceq $\operatorname{account}(U, \operatorname{del}$-account $(U, A))=\operatorname{account}(U, A)$ if $U=/=U^{\prime}$.

ceq $\operatorname{account}\left(U, \operatorname{deposit}\left(U^{\prime}, N, A\right)\right)=\operatorname{add}(N, \operatorname{account}(U, A))$ if $U==U^{\prime}$.

ceq $\operatorname{account}\left(U, \operatorname{deposit}\left(U^{\prime}, N, A\right)\right)=\operatorname{account}(U, A)$ if $U=/=U^{\prime}$.

ceq $\operatorname{account}\left(U\right.$, withdraw $\left.\left(U^{\prime}, N, A\right)\right)=\operatorname{add}(-(N)$, $\operatorname{account}(U, A))$ if $U==U^{\prime}$.

ceq $\operatorname{account}(U$, withdraw $(U, N, A))=\operatorname{account}(U, A) \quad$ if $U=/=U^{\prime}$.

\subsection{Synchronized Parallel Connection}

In this subsection, we add a user database (UserDB) to the bank account system example for having a more sophisticated user management mechanism. This enables query- 
ing whether an user already has an account in the bank account system. The users data base is obtained just by reusing (renaming) the set object of Section 12 The new account system compound object contains both synchronization patterns: broadcasting and client-server computing. add-account is related to add of Account by the projection operation for Account and it is also related to add of UserDB by the projection operation for UserDB. So, there is an overlapping of action groups (broadcasting). Also, add-account is related to add of Account by the projection operation for Account using the information of UserDB (client-server computing). The same holds for del-account. The following is the UML and CafeOBJ graphical representation of this:

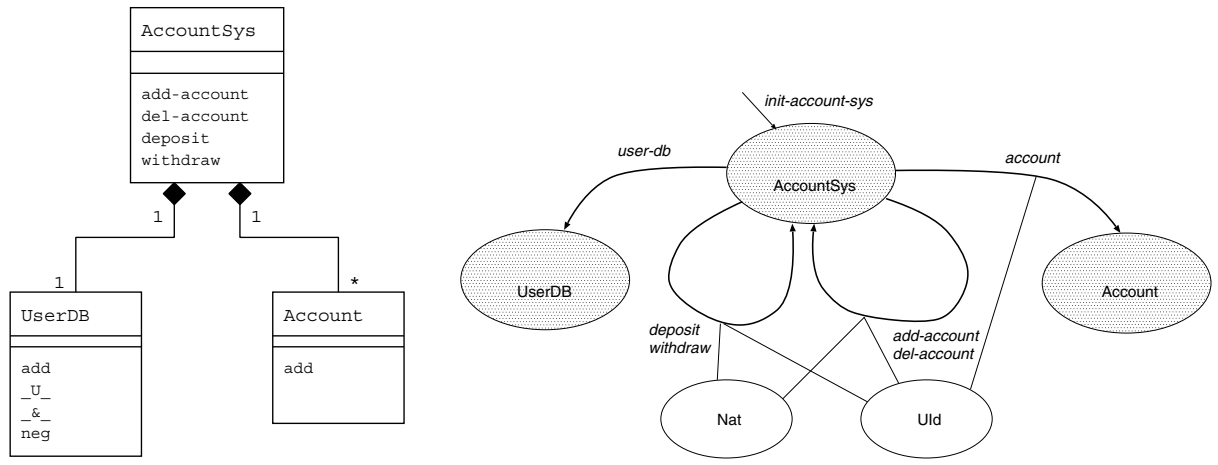

and here is the CafeOBJ code representing the equations for the projection operation for UserDB:

eq user-db(init-account-sys) = empty .

eq user- $d b($ add-account $(U, A S))=\operatorname{add}(U$, user- $d b(A S))$.

eq user-db(del-account $(U, A S))=n e g($ add $(U$, empty) $) \&$ user-db(AS) .

eq user-db(deposit( $U, N, A S))=$ user-db(AS) .

eq user- $d b$ (withdraw $(U, N, A S))=$ user- $d b(A S)$.

The following is the CafeOBJ code for the equations for the projection operation for Account, we skip here the equations of deposit and withdraw which are the same as in the previous example, and we also skip the equation for del-account which is similar to that of add-account:

eq account $(U$, init-account-sys $)=$ no-account .

ceq $\operatorname{account}(U$, add-account $(U, N, A S))=\operatorname{add}(N$, init-account $(U))$

if $U==U^{\prime}$ and not $(U$ in user-db(AS)).

ceq $\operatorname{account}(U, \operatorname{add}$-account $(U, N, A S))=\operatorname{account}(U, A S)$

if $U=/=U^{\prime}$ or $U$ in user- $d b(A S)$.

For add-account, we check whether the user is already registered and if not map it to add. If the user is already registered in UserDB, then skip.

It is interesting to mention that the same test red balance('u:Uld, add-account('u, 100, deposit('u, 30, add-account('u, 100, init-account-sys))))

gets different results in the previous account system example and in the current synchronized example due to the finer user management in the synchronized case. 


\subsection{Compositionality of Verifications}

In object-oriented programming, reusability of the source code is important, but in object-oriented specification, reusability of the proofs is also very important because of the verification process. We call this compositionality of verifications of components. In the CafeOBJ object composition method this is achieved by the following fundamental Theorem (for its proof see [8]):

Theorem 1. Given the states $s$ and $s^{\prime}$ of a compound object then the behavioural equivalence $\sim$ of the compound object is defined as follows:

$$
s \sim s^{\prime} \text { iff } \pi_{n}(s) \sim_{n} \pi_{n}\left(s^{\prime}\right) \text { for all } n \in O b j
$$

where $\mathrm{Obj}$ is a set of the labels for the components (you can consider these labels as the names of the component modules), $\pi_{n}$ is the projection operation corresponding to the component object $n$, and $\sim_{n}$ is the behavioural equivalence of the component.

Therefore, in the case of a hierarchic object composition, the behavioural equivalence for the whole system is just the conjunction of the behavioural equivalences of the base level objects, which are generally rather simple.

For example, the behavioural equivalence for the bank account system is a conjunction of the behavioural equivalence Account (indexed by the user identifiers) and UserDB, and these two are checked automatically by the CafeOBJ system. This means that behavioural proofs for the bank account system are almost automatic, without having to go through the usual coinduction process. Therefore, the behavioural equivalence _R[-__ of AccountSys can be defined by the following CafeOBJ code:

mod BEQ-ACCOUNT-SYSTEM \{ protecting(ACCOUNT-SYSTEM)

op _R[-]_ : AccountSys Uld AccountSys -> Bool

vars AS1 AS2 : AccountSys

var $U:$ Uld

eq $A S 1 R[U]$ AS2 $=\operatorname{account}(U, A S 1)={ }^{*}=\operatorname{account}(U, A S 2)$ and

user- $d b(A S 1)={ }^{*}=$ user- $d b($ AS2 $\left.).\right\}$

Notice the use of the parameterized relation for handling the conjunction indexed by the user identifiers.

Now, we will prove the true concurrency of withdrawals of two different users, which can be considered as a safety property for this system of bank accounts and which is formulated as the following commutativity behavioural property:

$$
\text { withdraw }(u 1, n 1 \text {, withdraw }(u 2, n 2, a s)) \sim \text { withdraw }(u 2, n 2, \text { withdraw }(u 1, n 1, a s))
$$

The following CafeOBJ code builds the proof tree containing all possible cases formed by orthogonal combinations of atomic cases for the users with respect to their membership to the user accounts data base. The basic proof term is TERM. The automatic generation of the proof tree (RESULT)is done by a meta-level encoding in CafeOBJ by using its rewrite engine for one-directional construction of the proof tree (this process uses the rewriting logic feature of CafeOBJ, hence the use of transitions (trans) rather than equations).

mod PROOF-TREE \{ protecting(BEQ-ACCOUNT-SYSTEM)

ops $n 1 n 2:->$ Nat -- arbitrary amounts for withdrawal 
ops u u1 u1' u2 u2' : -> Uld -- arbitrary user identifiers op as : -> AccountSys -- arbitrary state of the account system eq $u 1$ in user- $d b(a s)=$ true . -- first user is in the data base eq $u 2$ in user- $d b(a s)=$ true . -- second user is in the data base eq $u 1^{\prime}$ in user- $d b(a s)=$ false . -- first user is not in the data base eq $u 2^{\prime}$ in user- $d b(a s)=$ false . -- second user is not in the data base vars UU1 U2 : Uld op TERM : Uld Uld UId -> Bool -- basic proof term trans $\operatorname{TERM}(U, U 1, U 2)=>$ withdraw $(U 1, n 1$, withdraw $(U 2, n 2$, as)) $R[U]$

op TERM1: Uld Uld -> Bool withdraw(U2, n2, withdraw(U1, n1, as)).

trans $\operatorname{TERM1}(U, U 1)=>\operatorname{TERM}(U, U 1, \mathrm{U} 2)$ and $\operatorname{TERM}\left(U, U 1, \mathrm{U} 2^{\prime}\right)$.

op TERM2 : Uld -> Bool

trans $\operatorname{TERM2}(U)=>\operatorname{TERM1}(U, \mathrm{u} 1)$ and $\operatorname{TERM1}\left(U, \mathrm{u} 1^{\prime}\right)$.

op RESULT : -> Bool -- final proof term trans RESULT => TERM2(u1) and TERM2(u1') and TERM2(u) . \}

The execution of the proof term RESULT gives true after the system performs 233 rewrites.

\section{Conclusions and Future Work}

In this paper we presented the CafeOBJ object-oriented methodology for componentbased specification and verification which is based on the CafeOBJ behavioural abstraction paradigm. We also presented the basic behavioural specification methodology in CafeOBJ and gave a brief overview of the CafeOBJ language, system and specification environment.

Future work in this area will further explore and refine the current CafeOBJ methodologies exposed here with the aim of creating an industrial tool around these methodologies containing an industrial-oriented tutorial, a GUI interface probably based on the current CafeOBJ graphical notation, a graphical proof environment supporting distributed proofs over networks, etc.

Also, the power and sophistication of CafeOBJ gives the possibility to develop alternative behavioural specification and verification methodologies, including componentbased ones. We plan to study such alternative methodologies and their relationship to the current one.

\section{References}

[1] Rod Burstall and Joseph Goguen. The semantics of Clear, a specification language. In Dines Bjorner, editor, Proceedings, 1979 Copenhagen Winter School on Abstract Software Specification, pages 292-332. Springer, 1980. Lecture Notes in Computer Science, Volume 86.

[2] Manuel Clavel, Steve Eker, Patrick Lincoln, and Jose Meseguer. Principles of Maude. Electronic Notes in Theoretical Computer Science, 4, 1996. Proceedings, First International Workshop on Rewriting Logic and its Applications. Asilomar, California, September 1996.

[3] Răzvan Diaconescu. Category-based semantics for equational and constraint logic programming, 1994. DPhil thesis, University of Oxford. 
[4] Răzvan Diaconescu. Behavioural coherence in object-oriented algebraic specification. Technical Report IS-RR-98-0017F, Japan Advanced Institute for Science and Technology, June 1998. Submitted to publication.

[5] Răzvan Diaconescu. Extra theory morphisms for institutions: logical semantics for multiparadigm languages. J. of Applied Categorical Structures, 6(4):427-453, 1998.

[6] Răzvan Diaconescu and Kokichi Futatsugi. Logical foundations of CafeOBJ. 1998. Submitted to publication.

[7] Răzvan Diaconescu and Kokichi Futatsugi. CafeOBJ Report: The Language, Proof Techniques, and Methodologies for Object-Oriented Algebraic Specification, volume 6 of AMAST Series in Computing. World Scientific, 1998.

[8] Răzvan Diaconescu, Kokichi Futatsugi, and Shusaku Iida. Component-based algebraic specifications: - behavioural specification for component based software engineering -. In Behavioural Semantics of Object-oriented Business and System Specification. Kluwer, 1999.

[9] Răzvan Diaconescu, Joseph Goguen, and Petros Stefaneas. Logical support for modularisation. In Gerard Huet and Gordon Plotkin, editors, Logical Environments, pages 83-130. Cambridge, 1993. Proceedings of a Workshop held in Edinburgh, Scotland, May 1991.

[10] Kokichi Futatsugi, Joseph Goguen, Jean-Pierre Jouannaud, and Jose Meseguer. Principles of OBJ2. In Proceedings of the 12th ACM Symposium on Principles of Programming Languages, pages 52-66. ACM, 1985.

[11] Joseph Goguen and Rod Burstall. Institutions: Abstract model theory for specification and programming. Journal of the Association for Computing Machinery, 39(1):95-146, January 1992.

[12] Joseph Goguen and Răzvan Diaconescu. An Oxford survey of order sorted algebra. Mathematical Structures in Computer Science, 4(4):363-392, 1994.

[13] Joseph Goguen and Răzvan Diaconescu. Towards an algebraic semantics for the object paradigm. In Harmut Ehrig and Fernando Orejas, editors, Recent Trends in Data Type Specification, volume 785 of Lecture Notes in Computer Science, pages 1-34. Springer, 1994.

[14] Joseph Goguen and Grant Malcolm. A hidden agenda. Technical Report CS97-538, University of California at San Diego, 1997.

[15] Joseph Goguen and José Meseguer. Eqlog: Equality, types, and generic modules for logic programming. In Douglas DeGroot and Gary Lindstrom, editors, Logic Programming: Functions, Relations and Equations, pages 295-363. Prentice-Hall, 1986.

[16] Joseph Goguen and José Meseguer. Unifying functional, object-oriented and relational programming, with logical semantics. In Bruce Shriver and Peter Wegner, editors, Research Directions in Object-Oriented Programming, pages 417-477. MIT, 1987.

[17] Joseph Goguen and José Meseguer. Order-sorted algebra I: Equational deduction for multiple inheritance, overloading, exceptions and partial operations. Theoretical Computer Science, 105(2):217-273, 1992.

[18] Joseph Goguen, Timothy Winkler, José Meseguer, Kokichi Futatsugi, and Jean-Pierre Jouannaud. Introducing OBJ. In Joseph Goguen, editor, Algebraic Specification with OBJ: An Introduction with Case Studies. Cambridge. To appear.

[19] Rolf Hennicker and Michel Bidoit. Observational logic. In A. M. Haeberer, editor, Algebraic Methodology and Software Technology, number 1584 in LNCS, pages 263-277. Springer, 1999. Proc. AMAST'99.

[20] José Meseguer. Conditional rewriting logic as a unified model of concurrency. Theoretical Computer Science, 96(1):73-155, 1992.

[21] José Meseguer. Membership algebra as a logical framework for equational specification. In F. Parisi-Pressice, editor, Proc. WADT'97, number 1376 in Lecture Notes in Computer Science, pages 18-61. Springer, 1998. 